\title{
First do no harm: surrogate endpoints and the lesson of $\beta$-agonists in acute lung injury
}

\author{
Pradeep H Lakshminarayana' and Jeremy M Kahn,2,3 \\ University of Pittsburgh Department of Critical Care Medicine: Evidence-Based Medicine Journal Club, edited by Sachin Yende
}

\section{Expanded abstract Citation}

Matthay MA, Brower RG, Carson S, Douglas IS, Eisner M, Hite D, Holets S, Kallet RH, Liu KD, MacIntyre N, Moss M, Schoenfeld D, Steingrub J, Thompson BT: Randomized, placebo-controlled clinical trial of an aerosolized $\beta$-agonist for treatment of acute lung injury. National Heart, Lung, and Blood Institute Acute Respiratory Distress Syndrome (ARDS) Clinical Trials Network. Am J Respir Crit Care Med 2011, 184:561-568.

\section{Background}

$\beta_{2}$-Adrenergic receptor agonists accelerate resolution of pulmonary edema in experimental and clinical studies of acute lung injury (ALI).

\section{Methods \\ Objective: To determine whether an aerosolized $\beta_{2}$ - agonist would improve clinical outcomes in patients with ALI. \\ Design: Multi-center, phase III randomized, placebo- controlled clinical trial. \\ Setting: 33 hospitals participating National Heart, Lung, and Blood Institute Acute Respiratory Distress Syndrome (ARDS) Clinical Trials Network. \\ Subjects: Patients who were intubated and receiving mechanical ventilation, had bilateral infiltrates consistent with edema on frontal chest radiograph, had a ratio of $\mathrm{PaO}_{2}$ to $\mathrm{FIO}_{2}$ (fraction of inspired oxygen) of 300 or less, and not had clinical evidence of left atrial hypertension. A maximum enrolment of 1,000 patients was planned. Intervention: Patients were randomized to receive aerosolized albuterol $(5 \mathrm{mg}$ ) or saline placebo every 4 hours for up to 10 days.}

*Correspondence: kahnjm@upmc.edu

University of Pittsburgh School of Medicine, 602B Scaife Hall, 3550 Terrace Street,

University of Pittsburgh, Pittsburgh, PA 15261, USA

Full list of author information is available at the end of the article
Outcomes: The primary outcome variable was ventilatorfree days (VFD). Secondary outcome measures included mortality before hospital discharge on day 60 and day 90, the number of intensive care unit (ICU)-free days and the number of organ failure-free days.

\section{Results}

There were 282 patients enrolled before the trial was stopped for futility after the second interim analysis. The VFDs difference with albuterol treatment was unfavourable by -2.2 days, well past the futility boundary of -0.4 VFDs. VFDs were not significantly different between the albuterol and placebo groups (means of 14.4 and 16.6 days, respectively; 95\% confidence interval for the difference, -4.7 to 0.3 days; $P=0.087$ ). Rates of death before hospital discharge and the number of organ failure-free days were also not significantly different between the two groups. The number of ICU-free days was lower in the albuterol group in comparison with the placebo group (means of 13.5 and 16.2 days respectively; $95 \%$ confidence intervals for the mean difference, -4.9 to -0.4 days; $P=0.023)$. Overall, heart rates were significantly higher in the albuterol group by approximately 5 beats/minute in the first 2 days after randomization $(P<0.05)$, but rates of new onset atrial fibrillation $(10 \%$ in both groups) and other cardiac dysrhythmias were not significantly different.

\section{Conclusions}

These results suggest that aerosolized albuterol does not improve clinical outcomes in ALI patients. Routine use of $\beta_{2}$ agonist therapy in mechanically ventilated ALI patients cannot be recommended.

\section{Commentary}

Acute Lung Injury (ALI) is a major cause of respiratory failure and accounts for significant mortality in critically ill patients [1]. The pathophysiology of ALI involves a pro-inflammatory state with increased permeability and fluid leakage into the alveoli and interstitium, resulting in pulmonary edema and impaired gas exchange. The resolution of alveolar edema is a key factor in recovery 
from ALI and has therefore been the subject of a substantial number of experimental studies. Many of these studies demonstrate that transportation of sodium and chloride across the alveolar epithelial cells, which can be achieved by increased exogenous cyclic AMP stimulation with $\beta_{2}$ adrenergic agonists, is one of the principal mechanisms in alveolar fluid clearance [2]. In an ex vivo human lung model, alveolar fluid clearance doubled with use of a $\beta_{2}$ adrenergic receptor agonist [3]. Furthermore, a single center, phase II randomized trial (BALTI study) showed that patients with ALI receiving an intravenous $\beta_{2}$ adrenergic agonist had lower lung water, lower plateau airway pressures and lower Murray lung injury scores [4]. These laboratory and early clinical data showed promising results to suggest a potential therapeutic role of $\beta_{2}$ adrenergic agonists in improving clinical outcomes when used routinely in patients with ALI.

Based on this preliminary work, the Acute Respiratory Distress Syndrome (ARDS) Clinical Trials Network conducted the ALTA study [5]. ALTA was the first multicenter, phase III randomized study to test the role of routine use of a $\beta_{2}$ adrenergic agonist in improving clinical outcomes in patients with ALI. The primary outcome measure for this study was the number of ventilator free days (VFDs) by day 28. The trial was terminated prematurely after recruitment of only 282 patients of the proposed 1,000 patients at the second interim analysis on grounds of futility. The primary outcome, the number of VFDs to day 28, was not significantly different between study groups. Mortality before hospital discharge to days 60 and 90 and the number of organ failure-free days were also not significantly different between the two groups. The number of intensive care unit-free days was lower in the albuterol-treated group, suggesting that survivors receiving albuterol spent more time in the ICU than survivors receiving placebo. Also noted was that the albuterol-treated group had a higher heart rate and a tendency towards more fluid resuscitation. The KaplanMeier survivor curves suggested worse survival in patients who routinely received albuterol compared to placebo, although the statistical significance of this analysis was not reported.

There are several potential reasons why $\beta_{2}$ adrenergic agonists failed to improve outcomes in this study, despite promising physiological evidence. Among these are the possible lack of significant drug delivery to the aerated, nonedematous lung; the presence of injured alveolar epithelium unable to clear lung water; and down-regulation of $\beta_{2}$ adrenergic receptors with continued use of $\beta_{2}$ adrenergic agonist. Under these scenarios, the $\beta_{2}$ adrenergic agonists would fail to achieve their intended effect in real-life clinical settings.

Another possibility is that $\beta_{2}$ adrenergic agonist might successfully improve lung water clearance but harm patients in other ways. For example, $\beta_{2}$ adrenergic agonists cause tachycardia which might lead to greater fluid resuscitation in the study drug arm. This was seen in ALTA, where patients in the $\beta_{2}$ adrenergic agonist arm experienced higher heart rates and a trend towards higher cumulative fluid balance in the albuterol-treated arm, particularly on day 2 after randomization. $\beta_{2}$ adrenergic agonists might also lead to lactic acidosis and cardiac arrhythmias, as were noted in another recent randomized controlled trial stopped early for potential harm [6].

Along these lines, this clinical trial provides an important caveat to our use of surrogate endpoints to guide clinical therapy in the intensive care unit. Surrogate endpoints such as oxygenation and lung water measurements are valid only when they are causally-related to a patient-centered outcome (i.e. mortality), and there is no other link between the therapy of interest and the patient-centered outcomes [7]. Our field has been burned before, with numerous examples of therapies that improved surrogate outcomes but when studied in large trials ultimately were found to harm patients [8]. Of course, physiology is and will always be an important component of clinical decision making in the ICU. However, we must remember that when we make decisions based on physiological rationale alone, we may harm our patients.

With regards to $\beta_{2}$ adrenergic agonists in ALI, there is additional cause for concern. $\beta_{2}$ adrenergic agonists are among the most commonly prescribed medications for mechanically ventilated patients in the ICU. Often they are prescribed routinely, regardless of evidence of significant airflow obstruction or intrinsic positive endexpiratory pressure. In these cases, we may truly be causing harm in the absence of benefit. The ALTA study reminds us that no drug in the ICU is completely benign. Although this study certainly does not provide evidence precluding the use of inhaled $\beta_{2}$ adrenergic agonists in the ICU, any routine use in the absence of demonstrated airflow obstruction should be reconsidered.

\section{Recommendation}

Whenever we use surrogate endpoints and physiologic rationale to make decisions in patient care, investigators and clinicians should be cautious when translating the data into clinical benefit. As seen from this study, routine use of $\beta_{2}$ adrenergic agonist in ALI cannot be recommended, since there was no benefit and a suggestion of a tendency to cause harm to patients. $\beta_{2}$ adrenergic agonist therapy could be used as a therapeutic agent for use in patients with ALI and evidence of air flow obstruction, but not on a routine basis.

Competing interests

The authors declare that they have no competing interests. 


\section{Author details}

'Department of Critical Care Medicine, University of Pittsburgh, Pittsburgh, PA, USA. ${ }^{2}$ The Clinical Research, Investigation, and Systems Modeling of Acute Illness (CRISMA) Center, University of Pittsburgh, Pittsburgh, PA, USA. ${ }^{3}$ Department of Health Policy and Management, University of Pittsburgh Graduate School of Public Health, Pittsburgh, PA, USA

Published: 26 June 2012

\section{References}

1. Rubenfeld GD, Caldwell E, Peabody E, et al:. Incidence and outcomes of acute lung injury. N Engl J Med 2005, 353:1685-1693.

2. Matthay MA, Folkesson HG, Clerici C: Lung epithelial fluid transport and the resolution of pulmonary edema. Physiol Rev 2002, 82:569-600.

3. Sakuma T, Okaniwa G, Nakada T, et al:: Alveolar fluid clearance in the resected human lung. Am J Respir Crit Care Med 1994, 150:305-310

4. Perkins GD, McAuley DF, Thickett $D R$, et al.: The beta-agonist lung injury trial (BALTI): a randomized placebo-controlled clinical trial. Am J Respir Crit Care Med 2006, 173:281-287.
5. National Heart, Lung, and Blood Institute Acute Respiratory Distress Syndrome (ARDS) Clinical Trials Network, Matthay MA, Brower RG, et al:: Randomized, placebo-controlled clinical trial of an aerosolized beta(2)agonist for treatment of acute lung injury. Am J Respir Crit Care Med 2011 , 184:561-568.

6. Gao SF, Perkins GD, Gates $S$, et al:: Effect of intravenous beta-2 agonist treatment on clinical outcomes in acute respiratory distress syndrome (BALTI-2): a multicentre, randomized controlled trial. Lancet 2012, 379:229-235

7. Fleming TR, DeMets DL: Surrogate end points in clinical trials: are we being misled? Ann Intern Med 1996, 125:605-613.

8. Rubenfeld GD: Surrogate measures of patient-centered outcomes in critical care. Update in Intensive Care and Emergency Medicine, 2003.

doi:10.1186/cc11392

Cite this article as: Lakshminarayana PH, Kahn JM: First do no harm: surrogate endpoints and the lesson of $\beta$-agonists in acute lung injury. Critical Care 2012, 16:314. 